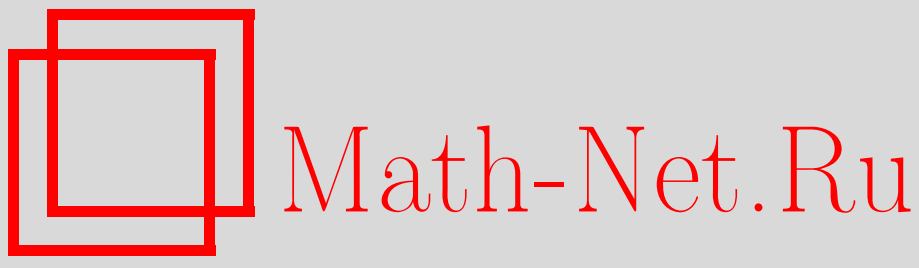

А. Р. Сафаров, О задаче сужения преобразования Фурье на гиперповерхности, Изв. вузов. Матем., 2019, номер 4, 66-72

DOI: https://doi.org/10.26907/0021-3446-2019-4-66-72

Использование Общероссийского математического портала Math-Net.Ru подразумевает, что вы прочитали и согласны с пользовательским соглашением

http://www . mathnet.ru/rus/agreement

Параметры загрузки:

IP: 3.80 .253 .173

26 апреля 2023 г., 09:12:53

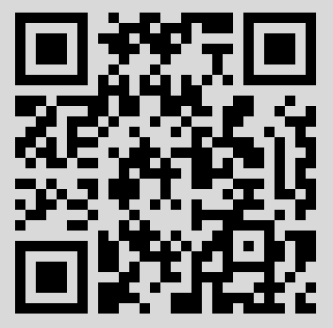


Известия вузов. Математика

2019 , № 4, c. $66-72$ https://kpfu.ru/science/nauchnye-izdaniya/ivrm

e-mail: izvuz.matem@kpfu.ru

\title{
A.P. CAФAPOB
}

\section{О ЗАДАЧЕ СУЖЕНИЯ ПРЕОБРАЗОВАНИЯ ФУРЬЕ НА ГИПЕРПОВЕРХНОСТИ}

\begin{abstract}
Аннотация. Работа посвящена ограничениям преобразования Фурье на модельных гиперповерхностях. Применение методов разложения Литтлвуда-Пэли позволяет получить точные оценки для показателя суммируемости. Эти оценки улучшают результаты А.Гринлифа для модельных гиперповерхности.
\end{abstract}

Ключевые слова: преобразование Фурье, суммируемые функции, задача сужения.

УДК: 517.518

DOI: 10.26907/0021-3446-2019-4-66-72

\section{ВВЕДЕНИЕ}

Пусть гладкая гиперповерхность в $\mathbb{R}^{n+1}$ с римановой поверхностной мерой $d S$ имеет конечный тип, т. е. каждая касательная гиперплоскость имеет конечный порядок касания с $S$. Рассмотрим плотность $d \mu:=\psi d S$ на $S$, где $0 \leq \psi \in C_{0}^{\infty}(S)$ - пространство бесконечногладких функций с компактным носителем.

Основная цель работы - найти максимальное значение $p$, для которого справедлива оценка

$$
\int_{S}|\widehat{f}(\xi)|^{2} \psi(\xi) d S(\xi) \leq C\|f\|_{\mathbb{L}^{p}\left(\mathbb{R}^{n+1}\right)}^{2}
$$

для любой функции $f \in C_{0}^{\infty}\left(\mathbb{R}^{n+1}\right)$, где $C_{0}^{\infty}\left(\mathbb{R}^{n+1}\right)$ - пространство бесконечно-гладких финитных, т. е. с компактным носителем, функций. Здесь $\widehat{f}(\xi)=\int_{\mathbb{R}^{n+1}} e^{-2 \pi i(x, \xi)} f(x) d x$ и $0 \leq \psi \in C_{0}^{\infty}(S)$ - фиксированная функция.

Эта задача называется проблемой об $\mathbb{L}^{p} \rightarrow \mathbb{L}^{2}$ ограничении преобразования Фурье [1]. Ограничение преобразования Фурье на нуль-мерных множествах имеет давнюю историю, начиная с классических результатов И.М. Стейна и П.А. Томаса (более подробно в [2], а также в [3]). Результаты работы [4] позволяют получить оценку снизу для более общего оператора ограничения.

Равномерные оценки преобразования Фурье мер позволяют получить оценку для $p$ ([1]). В этой работе методом разложения Литтлвуда-Пэли получено решение проблемы об ограничении преобразования Фурье суммируемых функций для некоторых гиперповерхностей. Эти оценки улучшают результаты А.Гринлифа [1] для модельных гиперповерхностей. В работе [5] получены оценки для преобразования Фурье в терминах многогранников Ньютона.

Поступила в редакцию 26.12.2017, после доработки 05.11.2018. Принята к публикации 19.12.2018

Благодарности. Работа выполнена при финансовой поддержке фонда фундаментальных исследований Республики Узбекистан, грант ОТ-Ф4-69. 
Пусть $S$ - гиперповерхность и $f \in \zeta\left(\mathbb{R}^{n+1}\right)$, где $\zeta\left(\mathbb{R}^{n+1}\right)$ - пространство Шварца, т. е. пространство бесконечно гладких быстро убывающих со всеми производными функций [6].

Основным результатом работы является

Теорема. Пусть $S$ - гиперповерхность, определеннал графиком функиии $\xi_{n+1}=\xi_{1}^{m_{1}} \cdots \xi_{l}^{m_{l}}$ в окрестности нуля, при этом $m_{1}+\cdots+m_{l} \geq 2$. Тогда для любой фиксированной неотрицательной функции $\psi \in C_{0}^{\infty}(S)$ при $p^{\prime} \geq 2\left(1+\max _{1 \leq j \leq l}\left\{m_{j}\right\}\right)$ существует $C_{p}$ такое, ито для любой функции $f \in \zeta\left(\mathbb{R}^{n+1}\right)$ справедлива оценка

$$
\left(\int_{S}|\widehat{f}(\xi)|^{2} \psi(\xi) d S(\xi)\right)^{1 / 2} \leq C_{p}\|f\|_{\mathbb{L}^{p}\left(\mathbb{R}^{n+1}\right)},
$$

где $p^{\prime}-$ сопряљсенное к р число, т.е. $\frac{1}{p}+\frac{1}{p^{\prime}}=1$.

Замечание 1. Если $l=1$ и $m=1$, то соответствующая гиперповерхность является гиперплоскостью и, следовательно, для любого $p>1$ аналогичное неравенство не имеет места. В этом случае условие $m_{1}+\cdots+m_{l} \geq 2$ теоремы также не выполняется.

В [7] получены точные оценки вида (2) в случае, когда $S \subset \mathbb{R}^{4}$ - гладкая поверхность коразмерности больше единицы, заданная в виде графика, т. е.

$$
S=\left\{\left(x_{1}, x_{2}, x_{3}, x_{4}\right): x_{3}=\phi_{1}\left(x_{1}, x_{2}\right), x_{4}=\phi_{2}\left(x_{1}, x_{2}\right)\right\},
$$

где $\phi_{1}, \phi_{2}$ - однородные полиномы степени $m_{1}$ и $m_{2}$, а также $m_{1} \geq m_{2} \geq 2$. При этом $\phi_{1}$, $\phi_{2}$ удовлетворяют некоторым условиям невырожденности. В нашем случае условия теоремы 1 ([7], с. 106) не выполняются.

В [8] получена связь между равномерными оценками и оценкой ограничения (2) для выпуклых гиперповерхностей конечно-линейного типа: если каждая прямая, лежащая на касательной гиперповерхности, имеет конечный порядок касания с гиперповерхностью. В нашем случае, вообще говоря, гиперповерхности не выпуклы и не удовлетворяют условиям конечности линейного типа.

\section{1. ВСПОМОГАТЕЛЬНЫЕ УТВЕРЖДЕНИЯ}

Приведем утверждения, необходимые для доказательства основной теоремы.

Лемма 1. Пусть $\phi(\xi)=\xi_{1}^{m_{1}} \xi_{2}^{m_{2}} \cdots \xi_{n}^{m_{n}}$, где $m_{j} \geq 1-$ натуральные числа, $m_{1}+m_{2}+$ $\cdots+m_{n} \geq 2$. Тогда для любого $\xi^{0} \in(\mathbb{R} \backslash 0)^{n}$ ранг матрици гессиана $\left(\frac{\partial^{2} \phi}{\partial \xi_{i} \partial \xi_{j}}\left(\xi_{1}^{0}, \xi_{2}^{0}, \ldots, \xi_{n}^{0}\right)\right)$ равняется $n$.

Доказательство. Если $n=1$ и, следовательно, $m_{1} \geq 2$, то утверждение тривиально. Достаточно рассмотреть гессиан $\frac{\partial^{2} \phi}{\partial \xi_{i} \partial \xi_{j}}$ в точке $(1,1, \ldots, 1)$. В противном случае через линейную замену переменных любую точку $\xi^{0} \in(\mathbb{R} \backslash 0)^{n}$ приведем к точке $(1,1, \ldots, 1)$. Непосредственные вычисления показывают, что

$$
\operatorname{det}\left(\frac{\partial^{2} \phi}{\partial \xi_{i} \partial \xi_{j}}(1,1, \ldots, 1)\right)=m_{1}^{2} m_{2}^{2} \ldots m_{n}^{2} \operatorname{det}(D+A),
$$

где $n \times n$-матрицы

$$
D:=\left(\begin{array}{ccccc}
-\frac{1}{m_{1}} & 0 & 0 & \ldots & 0 \\
0 & -\frac{1}{m_{2}} & 0 & \ldots & 0 \\
\ldots & \ldots & \ldots & \ldots & \ldots \\
0 & 0 & 0 & \ldots & -\frac{1}{m_{n}}
\end{array}\right), \quad A:=\left(\begin{array}{ccccc}
1 & 1 & 1 & \ldots & 1 \\
1 & 1 & 1 & \ldots & 1 \\
\ldots & \ldots & \ldots & \ldots & \ldots \\
1 & 1 & 1 & \ldots & 1
\end{array}\right)
$$


Так как $\operatorname{rang} D=n, \operatorname{rang} A=1$, то $\operatorname{dim}(\operatorname{Ker} A)=n-1$. Докажем, что $\operatorname{det}(D+A) \neq 0$.

Запишем $D+A=D\left(I+D^{-1} A\right)$, где $I-$ единичная $n \times n$-матрица. Так как ранг матрицы $D^{-1} A$ равен единице, то $\lambda_{1}=1$ является $(n-1)$-кратным собственным значением матрицы $I+D^{-1} A$. С другой стороны, $\lambda_{1}+\lambda_{2}+\cdots+\lambda_{n}=n-1+\lambda_{n}=\operatorname{tr}\left(I+D^{-1} A\right)=n-$ $\left(m_{1}+m_{2}+\cdots+m_{n}\right)$. Таким образом, $\lambda_{n}=1-\left(m_{1}+m_{2}+\cdots+m_{n}\right) \neq 0$. Следовательно, $\operatorname{det}\left(I+D^{-1} A\right)=1-\left(m_{1}+m_{2}+\cdots+m_{n}\right) \neq 0$.

Если $\phi$ имеет вид $\phi(\xi)=\xi_{1}^{m_{1}} \xi_{2}^{m_{2}} \ldots \xi_{l}^{m_{l}}$ (где $2 \leq l \leq n$ ) и $m_{j} \geq 1$, а также $m_{1}+m_{2}+\cdots+$ $m_{l} \geq 2$, то в точке $\xi^{0} \in(\mathbb{R} \backslash 0)^{n}$ матрица $D^{2} \phi\left(\xi^{0}\right)$ имеет ранг $l$.

Лемма 2. Пусть ф удовлетворяет условиям леммы 1. Для любого элемента $\eta$ функиия $\phi(\xi, \eta)=\phi(\xi)+(\eta, \xi)$ имеет лищь невырожденные критические точки при $1 / 2 \leq\left|\xi_{j}\right| \leq 2$, где $j=\overline{1, n}$, более того, если $\phi(\xi)=\xi_{1}^{m_{1}} \xi_{2}^{m_{2}} \ldots \xi_{l}^{m_{l}}$, то $\phi(\xi, \eta)=\phi(\xi)+\left(\eta^{0}, \xi\right)$ в точке $\xi^{0} \in(\mathbb{R} \backslash 0)^{n}$ имеет критическую точку ранга $l$, где $\eta^{0}=-\nabla \phi\left(\xi^{0}\right)$.

Доказательство. Для определенности рассмотрим случай $l=n$. Как отметили выше, применяя линейную замену переменных, можем считать, что $\xi^{0}=(1,1, \ldots, 1)$ - критическая точка фазовой функции. Из доказательства леммы 1 получим, что функция $\phi\left(\xi, \eta^{0}\right)$ в точке $\xi^{0}$ имеет невырожденную критическую точку, где $\eta^{0}=-\nabla \phi\left(\xi^{0}\right)$.

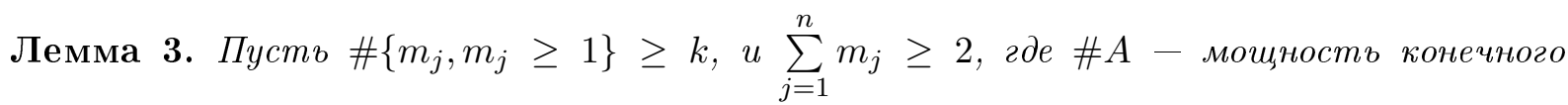
множества $A$. Тогда для любой фиксированной точки $\xi^{0} \in K \subset(\mathbb{R} \backslash 0)^{n}(K-$ некоторое компактное множество) существует окрестность $U\left(\xi^{0}\right)$ этой точки такал, что при любой функции $\psi \in C_{0}^{\infty}\left(U\left(\xi^{0}\right)\right)$ для преобразования Фуръе меры $\mu: \widehat{\mu}(\eta)=\int_{S} e^{i(\xi, \eta)} \psi(\xi) d S(\xi)$ справедлива оценка

$$
|\widehat{\mu}(\eta)| \leq C\|\psi\|_{C^{k}}(1+|\eta|)^{-k / 2}
$$

причем константа $C$ не зависит от $\xi^{0} \in K$, где $\|\psi\|_{C^{k}}-$ норма функции $\psi$ в пространстве $C^{k}(U(K))$; здесъ $U \subset(\mathbb{R} \backslash 0)^{n}$ - окрестность компакта $K$.

Лемма 3 вытекает из леммы 2 с применением леммы Морса ([9], с. 66) и доказывается стандартными методами анализа.

Доказательство. Пусть $-\eta=\lambda \omega, \lambda-$ неотрицательное вещественное число и $\omega \in S^{n}$ $\left(S^{n} \subset \mathbb{R}^{n+1}\right.$ - единичная сфера с центром в начале координат). Фиксируем $\omega=\omega^{0} \in S^{n}$. Если $\omega^{0}$ неколлинеарна единичной нормали $\nu_{0}$ к гиперповерхности $S$ в точке $\left(\xi^{0}, \phi\left(\xi^{0}\right)\right)$, то для фазовой функции

$$
\Phi(\xi, \eta)=\lambda\left(\omega_{1} \xi_{1}+\omega_{2} \xi_{2}+\cdots+\omega_{n} \xi_{n}+\omega_{n+1} \phi(\xi)\right)
$$

точка $\xi^{0}$ не является критической точкой. Следовательно, существуют окрестности $U\left(\xi^{0}\right)$ и $V\left(\omega^{0}\right)$ такие, что для произвольных точек $(\xi, \omega) \in U\left(\xi^{0}\right) \times V\left(\omega^{0}\right)$ имеем $\nabla_{\xi} \Phi(\xi, \eta) \neq 0$. Поэтому для произвольной амплитудной функции $\psi \in C_{0}^{\infty}\left(U\left(\xi^{0}\right)\right)$ с помощью формулы интегрирования по частям имеем оценку

$$
|\widehat{\mu}(\eta)| \leq C_{N}\|\psi\|_{C^{N}} / \lambda^{N}
$$

где $N$ - любое фиксированное натуральное число, $C_{N}$ - некоторое число, зависящее от $N$, $\|\psi\|_{C^{N}}$ - норма функции $\psi$ в пространстве $C^{N}\left(U\left(\xi^{0}\right)\right)$.

Пусть теперь $\omega^{0}$ коллинеарна к $\nu_{0}$, т. е. $\omega^{0}= \pm \nu_{0}$. Тогда $\nabla_{\xi} \Phi\left(\xi^{0}, \lambda \omega^{0}\right)=0$. В частности, имеем $\omega_{n+1}^{0} \neq 0$. Согласно лемме 2

$$
\omega_{n+1}^{0}\left(\frac{\omega_{1}}{\omega_{n+1}^{0}} \xi_{1}+\cdots+\frac{\omega_{n}}{\omega_{n+1}^{0}} \xi_{n}+\Phi(\xi)\right)
$$


в точке $\xi=\xi^{0}$ имеет критическую точку ранга $k$, т. е. ранг матрицы гессиана функции $\Phi(\xi)$ равен $k$. Согласно лемме Морса [9] существует семейство диффеоморфных отображений $g(\xi, \omega)$, определенных в достаточно малой окрестности $g: U\left(\xi^{0}\right) \times V\left(\omega^{0}\right) \rightarrow U(0) \times V(0) \subset$ $\mathbb{R}_{x}^{n} \times \mathbb{R}_{\sigma}^{n}$ такое, что справедливо соотношение

$$
\Phi\left(\xi\left(g^{-1}(x, \sigma)\right), \lambda \omega\left(g^{-1}(x, \sigma)\right)\right)=\lambda \omega_{n+1}^{0}\left( \pm x_{1}^{2} \pm x_{2}^{2} \pm \cdots \pm x_{k}^{2}+\Phi_{0}\left(x_{k+1}, \ldots, x_{n}, \sigma\right)\right) .
$$

Таким образом, применяя замену переменных $x=g(\xi, \omega)$, имеем

$$
\widehat{\mu}(\lambda \omega)=\int_{\mathbb{R}^{n}} \psi\left(g^{-1}(x, \sigma)\right)|J(x, \sigma)| e^{i \lambda \omega_{n+1}\left( \pm x_{1}^{2} \pm x_{2}^{2} \pm \cdots \pm x_{k}^{2}+\Phi_{0}\left(x_{k+1}, \ldots, x_{n}, \sigma\right)\right)} d x,
$$

где $J(x, \sigma)$ - якобиан замены переменных.

Наконец, применяя лемму Ван дер Корпута $k$ раз [2], получим

$$
|\widehat{\mu}(\lambda \omega)| \leq \frac{C\|\psi\|_{C^{k}}}{\lambda^{k / 2}} .
$$

Стандартные рассуждения о конечном покрытии единичной сферы $S^{n}$ завершают доказательство леммы.

\section{2. ДОКАЗАТЕЛЬСТвО ОсНОВНОГО РЕЗУЛЬТАТА}

Так как

$$
|\widehat{f}(\xi)|^{2}=\int_{\mathbb{R}^{n+1} \times \mathbb{R}^{n+1}} e^{-2 \pi i(x-y, \xi)} f(x) \bar{f}(y) d x d y,
$$

то интеграл (1) записывается в виде

$$
\int_{S}|\widehat{f}(\xi)|^{2} \psi(\xi) d S(\xi)=\int_{\mathbb{R}_{y}^{n+1}} \bar{f}(y) \int_{\mathbb{R}_{x}^{n+1}} f(x) \widehat{\mu}(y-x) d x d y,
$$

где $\widehat{\mu}(y-x)=\int_{S} e^{2 \pi i(y-x, \xi)} \psi(\xi) d S(\xi)$.

Обозначим через $T$ оператор свертки

$$
T f(y):=\int_{\mathbb{R}_{x}^{n+1}} f(x) \widehat{\mu}(y-x) d x .
$$

Если $T: \mathbb{L}^{p} \rightarrow \mathbb{L}^{p^{\prime}}$ - ограниченный оператор, то применяя неравенство Гёльдера, получим

$$
\left|\int_{\mathbb{R}_{y}^{n+1}} \bar{f}(y) T f(y) d y\right| \leq\|f\|_{\mathbb{L}^{p}}\|T f\|_{\mathbb{L}^{p^{\prime}}} \leq C\|f\|_{\mathbb{L}^{p}}^{2},
$$

где $C=\|T\|_{\mathbb{L}^{p} \rightarrow \mathbb{L}^{p^{\prime}}}$.

Далее, применяя методы [3], докажем ограниченность оператора $T$.

Пусть $y_{n+1}=\phi\left(y_{1}, \ldots, y_{n}\right)$, где $\phi(0)=0, \nabla \phi(0)=0$. Согласно теореме А. Гринлифа, если выполняется неравенство $|\widehat{\mu}(y)| \leq c /(1+|y|)^{r}$, то при $p^{\prime} \geq 2(1 / r+1)$ сверточный оператор ограничен как оператор $T: \mathbb{L}^{p} \rightarrow \mathbb{L}^{p^{\prime}}([1])$. В частности, если гауссова кривизна гиперповерхности $S$ нигде не обращается в нуль, то при $p^{\prime} \geq 2(2 / n+1)$ оператор свертки ограничен. Условие $p^{\prime} \geq 2(2 / n+1)$ эквивалентно условию $p \leq 2(n+2) /(n+4)$. Оператор свертки ограничен для таких гиперповерхностей и, следовательно, имеет место неравенство (1) при таких значениях $p$. Однако если выполняется неравенство

$$
|\widehat{\mu}(y)| \leq C \ln (2+|y|)^{k} /(1+|y|)^{r},
$$

где $k \geq 1$, то из результатов А.Гринлифа не следует ограниченность оператора $T$ при $p^{\prime}=2(1 / r+1)$, хотя следует ограниченность этого сверточного оператора при $p^{\prime}>2(1 / r+$ 1). Покажем, что для некоторых гиперповерхностей соответствующий оператор ограничен 
также при $p^{\prime}=2(1 / r+1)$. Для этой цели применим методы теории Литтлвуда-Пэли ([10], гл. 3).

Пусть $\chi \in C_{0}^{\infty}(\mathbb{R})$ - бесконечно-гладкая неотрицательная четная функция, удовлетворяющая условию

$$
\chi(x)=\left\{\begin{array}{lll}
1 & \text { при }|x| \leq 1 ; \\
0 & \text { при }|x| \geq 2,
\end{array} \quad \omega(x)=\chi(x)-\chi(2 x) .\right.
$$

Предположим, что $0 \leq \omega(x) \leq 1$.

Таким образом,

$$
\sum_{k=-\infty}^{\infty} \omega\left(2^{k} x\right)=1 \text { при всех } x \neq 0 .
$$

Более того, последний ряд сходится локально равномерно в $\mathbb{R} \backslash\{0\}([10])$. Аналогично имеем

$$
\sum_{k \in \mathbb{Z}^{n}} \omega\left(2^{k_{1}} x_{1}\right) \omega\left(2^{k_{2}} x_{2}\right) \cdots \omega\left(2^{k_{n}} x_{n}\right)=1
$$

для любого $x \neq 0$.

Введем обозначения

$$
\widehat{\Delta_{k} f}(\xi):=\omega\left(2^{k_{1}} \xi_{1}\right) \omega\left(2^{k_{2}} \xi_{2}\right) \cdots \omega\left(2^{k_{n}} \xi_{n}\right) \widehat{f}(\xi) \text { и } \bar{\sum}(x)=\left(\sum_{k \in \mathbb{Z}^{n}}\left|\Delta_{k} f(x)\right|^{2}\right)^{1 / 2} .
$$

Согласно теореме Литтлвуда-Пэли ([10], гл. 3) имеем

$$
c_{1}^{p}\|f\|_{\mathbb{L}^{p}} \leq\left\|\bar{\sum} f\right\|_{\mathbb{L}^{p}} \leq c_{2}^{p}\|f\|_{\mathbb{L}^{p}}
$$

где константы $c_{1}^{p}, c_{2}^{p}$ не зависят от функции $f \in \mathbb{L}^{p}\left(\mathbb{R}^{n}\right)$.

Пусть

$$
J_{k}(f):=\int_{S}|\widehat{f}(\xi)|^{2} \omega\left(2^{k_{1}} \xi_{1}\right) \omega\left(2^{k_{2}} \xi_{2}\right) \cdots \omega\left(2^{k_{n}} \xi_{n}\right) d S(\xi) .
$$

Запишем $J_{k}(f)$ в виде $n$-кратного интеграла

$$
J_{k}(f)=\int_{\mathbb{R}^{n}}\left|\widehat{f}\left(\xi^{\prime}, \phi\left(\xi^{\prime}\right)\right)\right|^{2} \omega\left(2^{k_{1}} \xi_{1}\right) \omega\left(2^{k_{2}} \xi_{2}\right) \cdots \omega\left(2^{k_{n}} \xi_{n}\right) \psi\left(\xi^{\prime}\right) \sqrt{1+\left|\nabla \phi\left(\xi^{\prime}\right)\right|^{2}} d \xi^{\prime},
$$

где $\xi^{\prime}=\left(\xi_{1}, \xi_{2}, \ldots, \xi_{n}\right)$ и $\phi\left(\xi^{\prime}\right)=\xi_{1}^{m_{1}} \xi_{2}^{m_{2}} \ldots \xi_{l}^{m_{l}}$.

Будем считать, что носитель $\psi$ достаточно мал и, следовательно, $k_{j} \geq 1$. В интеграле сделаем замену переменных $2^{k_{1}} \xi_{1}=\eta_{1}, \ldots, 2^{k_{n}} \xi_{n}=\eta_{n}$ и получим

$$
J_{k}(f)=2^{-|k|} \int_{\mathbb{R}^{n}}\left|\widehat{F}\left(\eta, \eta_{1}^{m_{1}} \cdots \eta_{l}^{m_{l}}\right)\right|^{2} \omega\left(\eta_{1}\right) \cdots \omega\left(\eta_{n}\right) \psi\left(2^{-k} \eta\right) \sqrt{1+\left|\nabla \Phi\left(2^{-k} \eta\right)\right|^{2}} d \eta
$$

где

$$
\widehat{F}\left(\eta, \eta_{1}^{m_{1}} \cdots \eta_{l}^{m_{l}}\right):=\widehat{f}\left(2^{-k} \eta, 2^{-(k, m)} \eta_{1}^{m_{1}} \cdots \eta_{l}^{m_{l}}\right) .
$$

Для

$$
F(x)=\int_{\mathbb{R}^{n+1}} e^{2 \pi i(\eta, x)} \widehat{F}(\eta) d \eta=\int_{\mathbb{R}^{n+1}} e^{2 \pi i(\eta, x)} \widehat{f}\left(2^{-k} \eta, 2^{-(k, m)} \eta_{n+1}\right) d \eta
$$

после замены переменных $2^{-k} \eta=\gamma, 2^{-(k, m)} \eta_{n+1}=\gamma_{n+1}$ имеем

$$
F(x)=2^{|k|+(k, m)} \int_{\mathbb{R}^{n+1}} e^{2 \pi i\left(2^{k} \gamma, x\right)+2^{(k, m)} \gamma_{n+1} x_{n+1}} \widehat{f}\left(\gamma, \gamma_{n+1}\right) d \gamma=2^{|k|+(k, m)} f\left(2^{k} x, 2^{(k, m)} x_{n+1}\right) .
$$


Итак,

$$
F(x)=2^{|k|+(k, m)} f\left(2^{k} x, 2^{(k, m)} x_{n+1}\right),
$$

где $|k|=k_{1}+\cdots+k_{l}$ и $(k, m)=k_{1} m_{1}+\cdots+k_{l} m_{l}$. Пусть

$$
K:=\left\{\eta:=\left(\eta_{1}, \ldots, \eta_{n}\right) \in R^{n}: 1 / 2 \leq\left|\eta_{j}\right| \leq 2, j=1, \ldots, n\right\} .
$$

Это компактное множество, содержащее носитель функции $\omega\left(\eta_{1}\right) \ldots \omega\left(\eta_{n}\right)$. Если $m_{1}, \ldots, m_{l} \geq 1$, то с учетом справедливости включения $K \subset(R \backslash\{0\})^{n}$ согласно лемме 3 для $p \leq \frac{2(l+2)}{l+4}$ получим

С другой стороны,

$$
J_{k}(f) \leq C 2^{-|k|}\|F\|_{\mathbb{L}^{p}\left(\mathbb{R}^{n+1}\right)} .
$$

$$
\begin{aligned}
\|F\|_{\mathbb{L}^{p}\left(\mathbb{R}^{n+1}\right)}^{2}=2^{2(|k|+(k, m))}\left(\int_{\mathbb{R}^{n+1}} \mid\right. & \left.\left.f\left(2^{k} x, 2^{(k, m)} x_{n+1}\right)\right|^{p} d x\right)^{2 / p}= \\
= & 2^{(|k|+(k, m))(2-2 / p)}\|f\|_{\mathbb{L}^{p}\left(\mathbb{R}^{n+1}\right)}^{2}=2^{2^{\frac{|k|+(k, m)}{p^{\prime}}}\|f\|_{\mathbb{L}^{p}\left(\mathbb{R}^{n+1}\right)} .}
\end{aligned}
$$

Следовательно,

$$
J_{k}(f) \leq 2^{-|k|+2 \frac{|k|+(k, m)}{p^{\prime}}}\|f\|_{\mathbb{L}^{p}\left(\mathbb{R}^{n+1}\right)} .
$$

Заметим справедливость следующих неравенств:

$$
\begin{gathered}
J_{k}(f) \leq \int_{\mathbb{R}^{n}}\left|\widehat{f}\left(\xi^{\prime}, \phi\left(\xi^{\prime}\right)\right)\right|^{2} \omega^{3}\left(2^{k_{1}} \xi_{1}\right) \cdots \omega^{3}\left(2^{k_{n}} \xi_{n}\right) \omega^{3}\left(2^{(k, m)} \phi\left(\xi^{\prime}\right)\right) \psi\left(\xi^{\prime}\right) \times \\
\times \sqrt{1+\left|\nabla \phi\left(\xi^{\prime}\right)\right|^{2}} d \xi^{\prime}=\int_{\mathbb{R}^{n}}\left|\widehat{f}\left(\xi^{\prime}, \phi\left(\xi^{\prime}\right)\right) \omega\left(2^{k_{1}} \xi_{1}\right) \cdots \omega\left(2^{k_{n}} \xi_{n}\right) \omega\left(2^{(k, m)} \phi\left(\xi^{\prime}\right)\right)\right|^{2} \times \\
\quad \times \omega\left(2^{k_{1}} \xi_{1}\right) \cdots \omega\left(2^{k_{n}} \xi_{n}\right) \omega\left(2^{(k, m)} \phi\left(\xi^{\prime}\right)\right) \psi\left(\xi^{\prime}\right) \sqrt{1+\left|\nabla \phi\left(\xi^{\prime}\right)\right|^{2}} d \xi^{\prime} \leq \\
\leq C 2^{-|k|+2 \frac{|k|+(k, m)}{p^{\prime}}}\left\|\Delta_{k,(k, m)} f\right\|_{\mathbb{L}^{p}\left(\mathbb{R}^{n+1}\right)}^{2} .
\end{gathered}
$$

Если $-|k|+2 \frac{|k|+(k, m)}{p^{\prime}} \leq 0$ или $p^{\prime} \geq 2\left(1+\frac{(k, m)}{|k|}\right)$, то для любого $k \in N^{n}$ имеем

$$
\begin{gathered}
J_{k}(f) \leq C\left\|\Delta_{k,(k, m)} f\right\|_{\mathbb{L}^{p}\left(\mathbb{R}^{n+1}\right)}^{2}, \\
J_{k}(f)=\int_{\mathbb{R}^{n}}|\widehat{f}(\xi)|^{2} \psi(\xi) \sqrt{1+|\nabla \phi(\xi)|^{2}} d \xi \leq \sum_{k \in \mathbb{N}^{n}} J_{k}(f) \leq \sum_{k \in \mathbb{N}^{n}}\left\|\Delta_{k} f\right\|_{\mathbb{L}^{p}\left(\mathbb{R}^{n+1}\right)}^{2} .
\end{gathered}
$$

Так как $p^{\prime}>2$, то $p<2$. Последняя оценка может быть записана в виде

$$
\begin{gathered}
\left(\int_{S}|\widehat{f}(\xi)|^{2} \psi(\xi) d S(\xi)\right)^{1 / 2} \leq\left(\sum_{k \in \mathbb{N}^{n}}\left\|\Delta_{k} f\right\|_{\mathbb{L}^{p}\left(\mathbb{R}^{n+1}\right)}^{2}\right)^{1 / 2}= \\
=\left(\sum_{k \in \mathbb{N}^{n}}\left(\int_{\mathbb{R}^{n+1}}\left|\Delta_{k} f(x)\right|^{p} d x\right)^{2 / p}\right)^{\frac{p}{2} \cdot \frac{1}{p}} \leq\left(\int_{\mathbb{R}^{n+1}}\left(\sum_{k \in \mathbb{N}}\left|\Delta_{k} f(x)\right|^{2}\right)^{p / 2} d x\right)^{1 / p} \leq c_{p^{p}}^{p}\|f\|_{\mathbb{L}^{p}\left(\mathbb{R}^{n+1}\right)} .
\end{gathered}
$$

В последней оценке использовали неравенство Минковского, т. е. если $f(x)$ - векторфункция со значением в нормированном пространстве, то справедливо неравенство

$$
\left\|\int_{A} f(x) d \mu\right\| \leq \int_{A}\|f(x)\| d \mu .
$$


Теорема доказана.

Заметим, что оценка $p^{\prime}>2\left(1+\max \left\{m_{l}\right\}\right)$ следует из результатов А.Гринлифа [1] с учетом [11] и [12]. Более того, если $m_{k}-$ различные натуральные числа, то оценка $p^{\prime} \geq$ $2\left(1+\max \left\{m_{l}\right\}\right)$ также следует из результатов А. Гринлифа [1]. Однако в случае, когда существуют $m_{k_{1}}, m_{k_{2}}$ такие, что $k_{1} \neq k_{2}$ и $m_{k_{1}}=m_{k_{2}}=\max \left\{m_{j}\right\}$, то результат А. Гринлифа дает лишь строгую оценку снизу. Таким образом, наши результаты позволяют получить оценку на границе $p^{\prime}=2\left(1+\max \left\{m_{l}\right\}\right)$ во всех случаях, уточняя оценку А.Гринлифа. Точность полученной оценки вытекает из неравенства снизу для соответствующей функции Кнапа [3].

Замечание 2. Аналогичные результаты справедливы для более общих гиперповерхностей вида $\xi_{n+1}=a(\xi) \xi_{1}^{m_{1}} \ldots \xi_{l}^{m_{l}}$, где $a(\xi)$ - гладкая функция с $a(0) \neq 0$.

В заключение автор выражает глубокую благодарность И.А.Икромову за постановку задачи и постоянное внимание к работе, а также рецензенту за ценные замечания.

\section{ЛитеРАтУРА}

[1] Greenleaf A. Principal curvature and harmonic analysis, Indiana Univ. Math. J. 30 (4), $519-537$ (1981).

[2] Stein E.M. Harmonic analysis. Princeton Univ. Press 13, Princeton, 1993.

[3] Ikromov I.A., Müller D. Fourier restriction for hypersurfaces in three dimensions and Newton polyhedra, Ann. Math. Stud. 194 (Princeton Univ. Press, Princeton and Oxford, 2016).

[4] Safarov A.R. On the $\mathbb{L}^{p}$-bound for trigonometric integrals, Anal. Math. J. 45 (1), 153-176 (2019).

[5] Magyar A. On Fourier restriction and Newton polygon, Proc. AMS. 137 (2), 615-625 (2009).

[6] Гельфанд И.М., Шилов Г.Е. Обобщеннъе функиии и действия над ними (ГИФМЛ, М., 1959).

[7] Laura De Carli and Alex Iosevich. Some sharp restriction theorems for homogeneous manifolds, Journal of Fourier Anal. and Appl. 4 (1), 105-128 (1998).

[8] Alex Iosevich Fourier transform, $\mathbb{L}^{2}$ restriction theorem, and scaling, Bolletino dell'Unione Math. Italiana ser. 8 2-B (2), 383-387 (1999).

[9] Федорюк М.В. Метод перевала (Наука, М., 1977).

[10] Стейн И., Вейс Г. Введение в гармонический анализ на евклидовых пространствах (Мир, М., 1974).

[11] Чубариков В.Н. О кратных рационалъных тригонометрических суммах и кратных интегралах, Матем. заметки 20 (1), 61-68 (1976).

[12] Сафаров А. Оценки некоторых кратных осцилляторных интегралов с полиномиалъной фазой, Узбекск. матем. журн. 2 106-116 (2012).

Акбар Рахманович Сафаров

Самаркандский государственный университет,

Университетский бульвар, д. 15, г. Самарканд, 140104, Республика Узбекистан,

e-mail : safarov-akbar@mail.ru

\section{A.R. Safarov}

\section{On a problem of restriction of Fourier transform on a hypersurface}

Abstract. This paper is devoted to the restriction problem of Fourier transform to model hypersurfaces. We show that the Littlewood-Paley methods allow to obtain sharp convervence estimate for the summability exponent. These estimates improve results by A. Greenleaf for model hypersurfaces.

Keywords: Fourier transform, summation functions, restriction problem.

Akbar Rakhmanovich Safarov

Samarkand State University, 15 Universitetskii blvd., Samarkand, 140104 Republic of Uzbekistan,

e-mail : safarov-akbar@mail.ru 